\title{
O PET-Saúde como instrumento de reorientação do ensino em Odontologia: a experiência da Universidade Federal do Espírito Santo
}

\author{
Caroline Marinho Gonçalves*, Karina Tonini dos Santos**, Raquel Baroni de Carvalho*** \\ * Graduada em Odontologia pela Ufes, Vitória - ES; ex-estagiária do \\ PET-Saúde \\ ** Doutora em Odontologia Preventiva e Social; Professora Adjunta \\ do Departamento de Medicina Social, Ufes, Vitória - ES; tutora do \\ PET-Saúde \\ *** Doutora em Odontologia; Professora Adjunta do Departamento de \\ Medicina Social, Ufes, Vitória - ES; tutora do PET-Saúde
}

\section{RESUMO}

As Diretrizes Curriculares Nacionais e a consolidação do SUS levaram as instituições de ensino superior em saúde no Brasil a reformular seus currículos, deixando de formar profissionais apenas tecnicistas para preparar profissionais voltados também para a realidade social, econômica, política e cultural do Brasil. O presente trabalho tem como objetivo discutir sobre o processo de reorientação do ensino em saúde/Odontologia, relatando a experiência do Programa de Educação pelo Trabalho para a Saúde (PETSaúde), iniciado na Ufes em 2010. O PET-Saúde foi criado pelo Ministério da Saúde em parceria com o Ministério da Educação, dando oportunidade aos acadêmicos de vivenciar o trabalho multidisciplinar dentro das unidades de saúde. As atividades extramuros propiciam um maior conhecimento por parte dos alunos que experimentam a estrutura dos serviços públicos de saúde, a participação no atendimento à população, a compreensão das políticas de saúde/ saúde bucal, o papel do cirurgião-dentista no serviço público, bem como o contexto social no qual futuramente o acadêmico poderá se inserir. O PET-Saúde oferece também um espaço de reflexão por meio das trocas de abordagens e experiências entre a realidade vivenciada pelos profissionais e os conhecimentos trazidos pelos alunos nos moldes acadêmicos. Conclui que, para a efetivação do SUS, os alunos dos cursos de saúde precisam de uma formação mais ampla, em ambientes diferenciados, com experiências inovadoras, atividades coletivas e não somente com a prática tradicional realizada em clínica de ensino de especialidades.

\section{DESCRITORES}

Educação superior. Educação em odontologia. Currículo. Estágio clínico.

D esde a década de 1940, o currículo do Curso de Odontologia esteve baseado nas recomendações de Flexner. Esse modelo de ensino caracteriza-se pela formação em ciências básicas nos primeiros anos de curso, diminuta ênfase aos aspectos de prevenção e promoção da saúde, atenção individualizada e aprendizagem enfocada para o ambiente clínico. ${ }^{11}$

As mudanças sociais ocorridas no Brasil e no mundo levaram à crise do modelo flexneriano tradicional e tecnocrata. ${ }^{15}$ No Brasil, o destaque à atenção primária ocorreu após a Reforma Sanitária Brasileira, que foi o ponto de partida para a implantação do Sistema Único de Saúde (SUS), o que levou as Instituições de Ensino em Saúde a avaliar a necessidade de desenvolver atividades extramuros, procurando articulação e integração com os serviços de saúde fora do campo universitário. ${ }^{1}$

De acordo com Garbin et al. ${ }^{13}$ (2006), o SUS resultou, em boa medida, da acumulação política e técnica propiciada pelas experiências da Medicina Comunitária e pela integração docente-assistencial (IDA), da década de 80 , definida por Marsiglia ${ }^{17}$ (1995) como união de esforços em um processo de crescente articulação entre instituições de educação 
e serviços de saúde adequados às reais necessidades da população, à produção de conhecimento e à formação de recursos humanos necessários em um determinado contexto da prática de serviços de saúde e de ensino.

O conceito de saúde explicitado na Constituição e os princípios que regeram a criação e implantação do SUS foram elementos fundamentais na definição das Diretrizes Curriculares Nacionais (DCN), ${ }^{2} \mathrm{em}$ 2002, propostas pela Lei de Diretrizes e Bases da Educação (LDB), o que garantiu uma integração efetiva entre o ensino e os serviços.

As DCNs sinalizam uma mudança na formação profissional do Curso de Graduação em Odontologia, na qual o aluno deve ser crítico, capaz de aprender a aprender, de trabalhar em equipe, de levar em conta a realidade social e, ainda, estipulam que $20 \%$ da carga horária plena do curso devem se caracterizar com estágio supervisionado. ${ }^{2}$

As atividades extramuros propiciam um maior conhecimento, por parte dos alunos, das estruturas dos serviços públicos de saúde, participação no atendimento à população, compreensão das políticas de saúde bucal, do papel do cirurgião-dentista (CD) e do contexto social no qual futuramente o acadêmico irá ingressar. ${ }^{20}$

Portanto, percebe-se a necessidade da formação do CD com um perfil voltado para a realidade social, econômica, política e cultural do Brasil. Dessa forma, a Universidade Federal do Espírito Santo (Ufes) aderiu ao Programa de Educação pelo Trabalho para a Saúde (PET-Saúde), regulamentado pela Portaria Interministerial $\mathrm{n}^{\circ} 421$, de 3 de março de 2010, inspirado no Programa de Educação Tutorial (PET), do Ministério da Educação, dando a oportunidade aos acadêmicos dos Cursos de Odontologia, Medicina, Enfermagem e Farmácia de vivenciar o trabalho multidisciplinar dentro das unidades de Saúde do SUS.

O presente trabalho tem como objetivo discutir sobre o processo de reorientação do ensino em saúde/Odontologia, relatando a experiência do Programa de Educação pelo Trabalho para a Saúde (PETSaúde), iniciado na Ufes em 2010.

\section{REVISÃo dE LITERATURA}

Atualmente, têm ocorrido significativos movimentos que criticam os modelos de formação profissional na área da saúde, principalmente na Medicina e na Enfermagem. Em relação à Odontologia, existe um atraso histórico desses movimentos de mudança, o que exige um grande esforço para que a saúde bu- cal seja integrada no novo contexto de ação interdisciplinar e multiprofissional. ${ }^{22}$

A maioria dos currículos de Odontologia está baseada em uma prática tecnicista, organizada em disciplinas isoladas, o que leva à formação de um profissional individualista, incapaz de interagir com a sociedade e solucionar seus problemas de saúde, dificultando sua inserção no mercado de trabalho. ${ }^{8}$ A Política de Educação e Desenvolvimento para o SUS também ressalta que

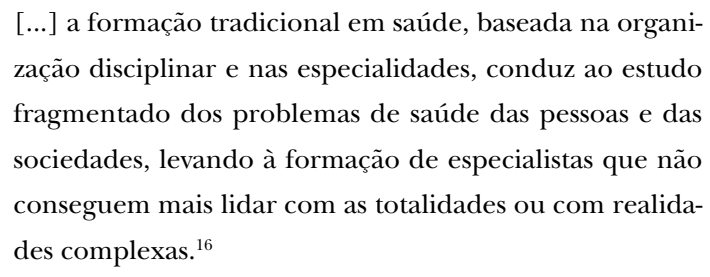

Pressionados pelas DCNs e pelo debate sobre qualidade e avaliação que se coloca em âmbito internacional, os Cursos de Odontologia começaram a buscar caminhos para a construção do projeto pedagógico, mudanças curriculares e profissionalização do trabalho docente. ${ }^{25}$

As DCNs assumem um papel estratégico no aperfeiçoamento do SUS, garantindo uma efetiva integração entre os conceitos de saúde e educação. ${ }^{22}$ As Diretrizes Curriculares para o Curso de Odontologia estabelecem o perfil do formando egresso como um profissional:

\footnotetext{
[...] capacitado ao exercício de atividades referente à saúde bucal da população, pautado em princípios éticos, legais e na compreensão da realidade social, cultural e econômica do seu meio, dirigindo sua atuação para a transformação da realidade em benefício da sociedade.
}

As diretrizes definem a realização de estágios supervisionados e programa de extensão, visto ser necessária a inserção do acadêmico no contexto social e a sua capacitação para

\footnotetext{
"[...] atuar com qualidade, eficiência e resolutividade, no Sistema Único de Saúde (SUS), considerando o processo da Reforma Sanitária Brasileira”. ${ }^{2}$
}

Diversos documentos apontam para a importância e necessidade do estabelecimento de estágios e convênios entre as instituições de ensino e os serviços de saúde como ferramenta imprescindível para a formação de recursos humanos. ${ }^{2,3}$ A Constituição Fede- 
O PET-Saúde como instrumento de reorientação do ensino em Odontologia: a experiência da Universidade Federal do Espírito Santo • Gonçalves CM, Santos KT, Carvalho RB

ral estabelece, em seu artigo 200, que:

“[...] ao Sistema Único de Saúde compete: III- ordenar a formação de recursos na área da saúde”. ${ }^{3}$

Para uma maior compreensão da rede de serviços como um espaço privilegiado de ensino, é preciso partir de um conceito ampliado de saúde na qual os cenários de aprendizagem são diversificados, agregando-se ao processo, além dos equipamentos de saúde, os equipamentos educacionais e comunitários. Portanto, é preciso sair das práticas profissionalizantes realizadas em clínicas de ensino de especialidades para as práticas de ensino em clínicas integradas e atividades extramurais em unidades do SUS. ${ }^{22}$

Dessa forma, as instituições de ensino superior vêm desenvolvendo atividades extramurais como parte integrante de seus cursos de graduação em saúde. Diversos estudos têm mostrado a efetividade dessas atividades, trazendo grandes perspectivas de inovação e concretização no que se refere à integração docente-assistencial. ${ }^{21}$ As atividades extramuros têm como base as ações de prevenção e promoção de saúde, sendo essa uma característica importante de sua filosofia de trabalho. ${ }^{7}$

É importante ressaltar que as ações de saúde não podem ser vistas dissociadas das questões referentes à habitação, saneamento básico, alimentação, meio ambiente, acesso a bens e serviços e situação econômica, como mostra a vivência proporcionada pelo estágio extramuro do Curso de Odontologia da Universidade Federal do Pará. Nesse espaço, a vivência dos acadêmicos consiste em conhecer a estrutura física, organização e planejamento de todas as atividades realizadas pela equipe de saúde na Casa da Família, bem como identificar, no espaço familiar, toda influência social e ambiental que envolve o processo saúde/doença na comunidade. Essa forma de estágio visa exatamente à formação de profissionais compromissados com a realidade e capazes de atender às necessidades da população em todos os níveis de atenção, influenciando na melhoria da saúde da comunidade em interação com as comunidades de acordo com os princípios do SUS. ${ }^{10}$

As normas do Conselho Federal de Odontologia $(2005)^{6}$ estabelecem que as atividades do estágio curricular deveriam ser realizadas sob a responsabilidade e coordenação direta de cirurgião-dentista, professor da instituição de ensino em que esteja o aluno matriculado. Entretanto, as recomendações da Abeno $(2002)^{9}$ indicam a possibilidade de o aluno desempe- nhar todas as atividades pertinentes a um profissional de saúde, com supervisão docente direta ou indireta, podendo haver preceptoria externa.

A grande maioria dos ex-alunos da Faculdade de Odontologia de Araçatuba (FOA/Unesp) afirmou que o serviço extramuro odontológico (Semo) desenvolvido nessa universidade foi um importante aprendizado, contribuindo para a sua formação profissional, além de ter sido uma forma de entrar em contato com o serviço público fora do ambiente da universidade. $^{19}$

Santa $\operatorname{Rosa}^{23}$ (2005) investigou a influência da disciplina Estágio Supervisionado na formação de estudantes do último período do Curso de Odontologia da UFMG. Os alunos relataram como uma das grandes vantagens do internato (e do serviço público) a oportunidade de trabalharem em equipe. Referiramse, ainda, à importância da interação com outras áreas, como uma oportunidade de aumentar conhecimentos, trocar experiências e crescer profissionalmente, permitindo um atendimento mais humano e integral ao paciente. Os alunos se surpreenderam com a constatação de que o serviço público funciona, apesar de suas limitações.

As atividades extramuros têm sido utilizadas com sucesso entre os graduandos dos cursos da saúde (Odontologia, Nutrição, Enfermagem, Farmácia e Fisioterapia) da Universidade Paulista, Campus Vargas de Ribeirão Preto, por meio do exercício de práticas educativas e preventivas na comunidade, em parcerias públicas e privadas. Nessa experiência, observouse uma grande interação entre os alunos, em que um respeitava e se interessava pelo trabalho do outro. Os alunos de Farmácia queriam aprender a melhor técnica para escovar os dentes; os alunos da Enfermagem solicitaram uma tabela de calorias; os alunos de Odontologia estavam preocupados com a postura. ${ }^{12}$

Mendes et al. ${ }^{18}$ (2006) mostraram que, pelo convênio firmado entre a Universidade Federal do Piauí (UFPI) e a Prefeitura Municipal de Teresina, foi oportunizada aos estudantes do estágio supervisionado a inserção em atividades desenvolvidas por equipes de saúde bucal do PSF. Essa experiência familiarizou e capacitou os estudantes a trabalharem na realidade que enfrentarão no mercado de trabalho. Além disso, os atendimentos extramurais dão abertura a novos campos para o desenvolvimento de pesquisas e ao ensino que ali se constroem, por meio de trocas de abordagens e experiências entre os profissionais que atuam no serviço público e os alunos que trazem o conhecimento nos moldes acadêmicos. 


\section{RELATO DA EXPERIÊNCIA: PET-SAÚdE - UNIVERSIDADE FEDERAL DO ESPÍRITO SANTO (UFES)}

Apenas recentemente, o Ministério da Saúde tem se preocupado em orientar o processo de formação dos recursos humanos da área, estabelecendo para tanto parceria com o Ministério da Educação. ${ }^{22}$ Dessa parceria surgiu o PET-Saúde, um programa destinado a viabilizar o aperfeiçoamento e a especialização em serviço, bem como a iniciação ao trabalho, estágios e vivências, dirigidos, respectivamente, aos profissionais e estudantes da área da saúde, conforme as necessidades do SUS.

A Ufes, de acordo com a Portaria Interministerial do MS/MEC $\mathrm{n}^{\circ} 18$, de 16 de setembro de 2009, disponibilizou, inicialmente, 72 vagas para a participação de seus estudantes no referido projeto como monitores. As vagas foram distribuídas para quatro cursos de graduação:

- 24 vagas para Enfermagem,

- 12 para Farmácia,

- 24 para Medicina e

- 12 para Odontologia.

Os alunos foram selecionados por meio de prova escrita, entrevista e análise do histórico escolar (como critério de desempate).

O programa tem como objetivos específicos: estabelecer o processo de aprendizagem dos estudantes dos cursos supracitados da Universidade Federal do Espírito Santo em um cenário de práticas, no âmbito da Estratégia de Saúde da Família, nas Unidades de Saúde da Região de Maruípe, no município de Vitória; promover o desenvolvimento de pesquisa aplicada no campo de abrangência da Estratégia de Saúde da Família, integrando o corpo técnico da Secretaria Municipal de Saúde (Semus) e o corpo docente da Ufes; fomentar a aprendizagem no contexto da multidisciplinaridade, da interdisciplinaridade e da transdisciplinaridade no cenário de práticas representado pelas Unidades de Saúde da Região de Maruípe, no município de Vitória; fomentar a estruturação do Núcleo de Excelência Clínica Aplicada à Atenção Básica no município de Vitória.

Os acadêmicos foram distribuídos em grupos multidisciplinares para atuar nas seguintes Unidades de Saúde da Família de Vitória:

- Thomaz Tommasi (Bonfim),

- Michel Minassa (Maruípe),

- Benedito Gomes da Silva (Santa Marta),

- Luiz Cláudio Passos (Andorinhas),

- Maria Rangel dos Santos (Consolação) e

- Dr. Gilson Santos (Bairro da Penha).
Posteriormente, foi incluída no Programa mais uma equipe do Curso de Odontologia. Assim, seus componentes são:

a) dois tutores constituídos por docentes odontólogas do Departamento de Medicina Social da UFES;

b) doze preceptores constituídos por odontólogos das equipes das Unidades de Saúde da Família: Michel Minassa (Maruípe), Dr. Gilson Santos (Bairro da Penha), Luiz Cláudio Passos (Andorinhas), Benedito Gomes da Silva (Santa Marta) e Maria Rangel dos Santos (Consolação);

c) vinte e quatro estudantes monitores escolhidos entre aqueles que estavam entre o quinto e o oitavo período do curso, os quais apresentam inserção de oito horas por semana nas atividades do Programa;

d) trinta e seis estudantes do sexto e sétimo período do curso, que estejam inseridos nas Equipes de Saúde da Família desenvolvendo prática supervisionada ou estágio curricular regular. Esses estudantes devem cumprir carga horária mínima de cinco horas diárias nas equipes de Saúde da Família das Unidades Básicas de Saúde.

Todos os estudantes estão integrados às equipes, participando das atividades de atenção primária. Adquirem vivência em visitas domiciliares, atendimento básico em Odontologia, ações educativas, e em acolhimento e reuniões de equipe. Além disso, os estudantes monitores são responsáveis, em conjunto com os de Medicina, os de Farmácia e os de Enfermagem, pela condução longitudinal do Plano de Pesquisa, com a participação dos estudantes em prática supervisionada ou estágio curricular.

Assim, a supervisão do estágio se dá em diferentes níveis. O docente/tutor tem como função principal a orientação do aluno no planejamento, execução e avaliação pessoal e de desempenho, incentivando-o e abrindo-lhe horizontes nas formas de aplicação dos conhecimentos adquiridos em sala de aula. Os docentes responsáveis pela supervisão não estão, necessariamente, em todos os locais do estágio, mas sempre devem estar disponíveis para os alunos.

Os preceptores acompanham o trabalho diário rotineiro desenvolvido pelos alunos, visando ao esclarecimento de dúvidas e aplicação de conhecimentos teórico-práticos, de acordo com o campo do estágio. Eles devem possibilitar a atuação do aluno, assumindo com a Universidade a responsabilidade pelo processo de ensino-aprendizagem, em um trabalho de parceria.

Os resultados das ações em saúde são avaliados com os participantes, mediante a discussão das ativi- 
dades desenvolvidas. Os acadêmicos são avaliados mensalmente, em função de seu envolvimento em todas as atividades propostas. É fundamental a participação em eventos científicos, divulgando os resultados do projeto.

O Plano de Pesquisa, que apresenta como tema "Padrão de comportamento relacionado à saúde de adolescentes residentes na região de Maruípe em Vitória, Espírito Santo", tem caráter multidisciplinar, com inserção de todos os cursos simultaneamente. Dessa forma, a pesquisa tem como objetivo conhecer o padrão de comportamento e as necessidades de saúde dos adolescentes de 15 a 19 anos, residentes nos bairros da região de Maruípe, a fim de elaborar estratégias de prevenção e assistência direcionadas a essa população.

A amostra da pesquisa foi obtida por meio do cadastro, feito pela ESF, de todos os adolescentes nessa faixa etária, residentes na região citada. Foi realizada uma seleção aleatória simples dos adolescentes que participarão do estudo. O tamanho da amostra foi determinado com base na estimação da prevalência de DST (Chlamydia trachomatis) em adolescentes de 15 a 19 anos. No estudo anterior, a prevalência observada foi de 11,4\% entre as adolescentes sexualmente ativas. Considerando-se uma variação de $3 \%$, a amostra a ser estudada seria de 357 adolescentes. Tendo em vista que se estima que, aproximadamente, $30 \%$ dos adolescentes, nessa faixa etária, não têm atividade sexual, ${ }^{5} \mathrm{o}$ tamanho final da amostra está sendo calculado em 464 adolescentes.

O questionário utilizado foi baseado no questionário da Analyse des Comportements Sexuels des Jeunes $(\mathrm{ACSJ})^{14} \mathrm{e}$ validado no estudo piloto. Foi aplicado pelos estudantes treinados para tal e contém:

- dados sociodemográficos (idade, escolaridade, religião, profissão, estado civil e dados sobre a família);

- dados clínicos (sintomas DST, contracepção, gravidez e abortos);

- sexuais (carícias, beijos, relações não sexuais, primeira relação sexual);

- sobre comportamentos de risco (uso de preservativos, número de parceiros sexuais, prostituição, uso de álcool e drogas, transfusão de sangue) para infecção pelo HIV e outras DSTs;

- conhecimentos sobre contracepção e

- autopercepção sobre a saúde bucal.

Além do referido Plano de Pesquisa, os monitores, em conjunto com os preceptores e tutores, têm a oportunidade de realizar, na unidade onde atuam, pesquisas sobre diversos temas relativos à Atenção Primária.

\section{DISCUSSÃo}

É visível que mudanças estão ocorrendo no ensino superior do Brasil, pois a rigidez dos currículos mínimos deixou de existir, dando espaço aos currículos flexíveis, de acordo com a realidade. ${ }^{24}$ Essas mudanças se devem à consolidação do SUS e, principalmente, à expansão do PSF, os quais levaram muitas instituições de ensino superior a repensar os seus currículos, a fim de formar profissionais que atendam às necessidades do país e, assim, melhorar os índices de saúde. ${ }^{15} \mathrm{O}$ aluno, em contato com comunidades carentes, além de aprendizado, exerce cidadania, tornando-se um profissional mais humano. ${ }^{12}$

De acordo com Morita e Kriger (2003), ${ }^{22}$ o SUS constitui um significativo mercado de trabalho para os profissionais da Odontologia, principalmente com a inserção da saúde bucal na Estratégia de Saúde da Família. O PET-Saúde na UfeS proporciona aos acadêmicos vivenciar as atividades desenvolvidas pelos profissionais inseridos na ESF, oportunizando que eles relacionem a teoria com prática e conheçam, de perto, como é o trabalho na rede pública de saúde. Além disso, a ESF é uma oportunidade única de resgatar uma prática mais humanista, em que o profissional cria um vínculo de responsabilidade com o paciente.

O estágio no PET-Saúde propicia aos alunos de Odontologia da Ufes o conhecimento das estruturas organizacional, administrativa, gerencial e funcional dos serviços públicos de saúde. Possibilita sua participação no atendimento à população, a compreensão das políticas de saúde bucal e do papel do cirurgiãodentista, o conhecimento das bases epidemiológicas do método clínico e de suas aplicações práticas nos programas de saúde bucal. Também oferece o conhecimento dos parâmetros e/ou instrumentos de planejamento utilizados nos projetos de saúde e programas de saúde bucal. ${ }^{26}$

A função da universidade deve ser a de identificar corretamente os problemas de saúde de cada município ou região e dizer como resolvê-los. Assim, o ensino e a pesquisa devem ser direcionados para ações de impactos sociais que possibilitem melhores condições de vida para a população. ${ }^{13}$ É exatamente essa proposta que o plano de pesquisa do PET-Saúde está desenvolvendo. O objetivo é conhecer como os adolescentes do município de Maruípe se comportam e quais são as suas necessidades, para, então, serem elaboradas estratégias de prevenção e assistência direcionadas e 
essa faixa etária. Vale ressaltar que essa demanda surgiu após muita discussão com os representantes da Secretaria Municipal de Saúde de Vitória.

Sabe-se que muitas são as dificuldades a serem vencidas para que o PET-Saúde realmente alcance seus objetivos, dentre elas:

- Por parte dos alunos

- o significado do SUS pode não ser claro;

- a falta de conhecimento sobre o significado e a importância do trabalho multidisciplinar;

- o não cumprimento de todas as atividades exigidas mensalmente;

- Por parte dos docentes

- pouco conhecimento e falta de conscientização de alguns professores do Curso de Odontologia sobre o SUS, não valorizando, às vezes, a importância do projeto;

- desconhecimento dos docentes da possibilidade de realização de pesquisas no SUS;

- Por parte dos usuários

- falta de colaboração dos pais dos adolescentes para que autorizem seus filhos a participar da pesquisa;

- pouco interesse dos adolescentes na pesquisa para que respondam de forma sincera ao extenso questionário.

O desafio que está posto é muito grande, porém espera-se que não faltem empenho e esforços dos docentes e dos alunos da Ufes, a fim de se garantir uma melhor qualificação, pois, como ocorreu nas experiências relatadas, o estágio supervisionado ocasionará bons resultados na formação profissional não só do ponto de vista técnico-científico, mas, acima de tudo, do ser humano e do cidadão socialmente comprometido. A interação ativa do aluno com a população e profissionais de saúde deve ocorrer desde o início do processo de formação, trabalhando com problemas reais e assumindo responsabilidades com grau de complexidades crescentes.

\section{CONSIDERAÇÕES FINAIS} que:

Após relatar a experiência, é possível considerar a) os alunos que, durante a graduação, participam de atividades extramuros, estarão, com certeza, mais bem preparados para atuar na comunidade, sobretudo em serviços de saúde, por terem a oportunidade do convívio e da concepção de uma consciência social, conhecendo as diferenças econômicas e culturais da população;

b) quando atividades extramuros são realizadas, contando com a participação de estudantes de outros cursos da saúde, o espírito de equipe e a capacidade de troca de informações com diferentes indivíduos ocasionam uma atenção integral mais humanizada do paciente e reforçam o que preconizam as Diretrizes Curriculares Nacionais;

c) para a efetivação do SUS, os alunos dos cursos de saúde precisam de uma formação mais ampla, em ambientes diferenciados, com experiências inovadoras, atividades coletivas e não somente a prática tradicional realizada em clínica de ensino de especialidades.

\section{ABSTRACT}

"PET-Saúde" as an instrument to provide dentistry teaching with new guidelines: the experience of the Federal University of Espírito Santo

The National Curricular Guidelines and the consolidation of the Unified Health System (SUS) have led higher education health institutions in Brazil to restructure their curriculum with the objective of preparing professionals not only technically but also to meet Brazil's social, economic, political and cultural challenges. The aim of the present study was to discuss the process of providing health/dentistry teaching with new guidelines, by reporting an experience gained in the "Programa de Educação pelo Trabalho para a Saúde - PET-Saúde" (Education Program through Health Work), launched at Ufes in 2010. "PET-Saúde" was created by the Ministry of Health in partnership with the Ministry of Education and gives students the opportunity of experiencing the multidisciplinary work conducted in Health Centers. The extramural activities give students broader knowledge of how public health services are structured, hands-on experience in treating the population, and a better understanding of the health/oral health policies, of the role of the dentist in public services, and of the social context in which the student will eventually be involved. "PETSaúde" also encourages reflection through the exchange of approaches and experiences between the real-world practice experienced by professionals and the knowledge brought by students from the academic world. In conclusion, in order for SUS to be put into place effectively, students of health courses need broader preparation and training in wide-ranging environments, and not only the experience of traditional practice offered by clinics teaching specialties, but also innovative experiences and collective activities. 
O PET-Saúde como instrumento de reorientação do ensino em Odontologia: a experiência da Universidade Federal do Espírito Santo • Gonçalves CM, Santos KT, Carvalho RB

\section{DESCRIPTORS}

Education, higher. Education, dental. Curriculum. Clinical clerkship. "

\section{REFERÊNCIAS}

1. Botti, MRV. Aprender fazendo: os caminhos da extensão universitária [Tese]. Santa Maria: Curso de Odontologia da Universidade Federal de Santa Maria; 1993.

2. Brasil. Conselho Nacional de Educação. Diretrizes Curriculares Nacionais do Curso de Graduação em Odontologia. Resolução no CNE/CES 3/2002. Diário Oficial da União, Brasília, 4 mar. 2002. Seção 1, p. 10.

3. Brasil. Constituição da República Federativa do Brasil. 12. São Paulo: Rideel; 2006. 341 p.

4. Brasil. Ministério da Saúde/Secretaria de Gestão do Trabalho e da Educação na Saúde. Caminhos para as mudanças da formação e desenvolvimento dos profissionais de saúde: diretrizes para a ação política para assegurar educação permanente no SUS. Brasília, maio 2003. (mimeografado)

5. CEBRAP (Centro Brasileiro de Análise e Planejamento). Comportamento sexual da população brasileira e percepções sobre HIV e AIDS. Brasília: Ministério da Saúde, SPS/PN/DST/ AIDS, 2000. 248 p.

6. Conselho Federal de Odontologia (CFO). Consolidação das normas para procedimentos nos Conselhos de Odontologia. Resolução 63/2005 de 2005

7. Costa ICC, Unfer B, Oliveira AGRC, Arcieri RM, Saliba NA. Integração universidade-comunidade: análise de atividades extramurais em odontologia nas universidades brasileiras. Rev Cons Reg Odontol Minas Gerais 2000; 3(6): 146-53.

8. Dias MC, Presta AA, Gava-Simioni L, Souza PB, Saliba NA. Currículo inovador em odontologia: considerações gerais. Saúde em Debate, Rio de Janeiro, 2005; 29 (69): 72-7.

9. Diretrizes da Associação Brasileira de Ensino Odontológica (ABENO) para definição do estágio supervisionado nos Cursos de Odontologia. In: Reunião paralela ao $20^{\circ}$ Congresso Internacional de São Paulo, APCD, São Paulo, 29/01/02.

10. Emmi DT, Cardoso DG, Araújo IC, Araújo MVA. Estágio Extramural do Curso de Odontologia da UFPA nas Unidades de Saúde do município de Belém-Pará. Medcenter, 2007.

11. Feuerwerker L, Marsiglia R. Estratégias para mudanças na formação de RHs com base nas experiências IDA/UNI. Divulgação em Saúde Para Debate, Rio de Janeiro, 1996;(12):24-8.

12. Galassi MAS, Barbin EL, Spanó JCE, Melo JAJ, Tortamano N, Perri de Carvalho AC. Atividades extramuros como estratégia viável no processo ensino-aprendizagem. Revista da ABENO, 2006;6(1):66-9.

13. Garbin CAS, Saliba NA, Moimaz SAS, Santos KT. O papel das universidades na formação de profissionais na área de saúde. Revista da ABENO, 2006; 6(1): 6-10.
14. Lagrange H, Lhomond B. L'entrée dans la sexualité: lé comportement des jeunes dans lé contexte du SIDA. Paris: La Découvert (Recherches), 1997. 431 p.

15. Lazzarin HC, Nakama L, Júnior LC. O papel do professor na percepção dos alunos de odontologia. Saúde e Sociedade, 2007; 16(1): 90-101.

16. Markert, Werner. Novos paradigmas do conhecimento e modernos conceitos de produção: implicações para um nova didática na formação profissional. Educação e Sociedade, 2000; 21(72): 177-96.

17. Marsiglia RMG, Relação ensino/serviços: dez anos de integração docente assistencial (IDA) no Brasil. São Paulo: Editora Hucitec; 1995. 124 p.

18. Mendes RF, Moura MS, Prado Júnior RR, Moura LFAD, Lages GP, Gonçalves MPR. Contribuição do Estágio Supervisionado da UFPI para formação humanística, social e integrada. Revista da ABENO, 2006; 6(1): 61-5.

19. Moimaz SAS, Saliba NA, Arcieri RM, Garbin CAS, Saliba O, Zina LG. Percepção de ex-alunos sobre a contribuição do Serviço Extramuro Odontológico (SEMO) da FOA-UNESP na formação profissional. Cienc Exten 2004; 1(2): 149-62.

20. Moimaz SAS, Saliba NA, Garbin CAS, Zina LG, Furtado JF, Amorim JA. Serviço Extramuro Odontológico: impacto na formação profissional. Pesq. Bras. Odontopediatria Clín. Integr. 2004; 4(1): 53-7.

21. Moimaz SAS, Saliba NA, Garbin CAS, Zina LG. Atividades extramuros na ótica de egressos do Curso de Graduação em Odontologia. Revista da ABENO, 2008; 8(1): 23-9.

22. Morita MC, Kriger L. Mudanças nos cursos de Odontologia e a interação com o SUS. Revista da ABENO, 2004; 4(1): 17-21.

23. Santa Rosa, Talhita Tryrza de Almeida. A influência do Estágio Supervisionado na formação de estudantes do Curso de Odontologia da UFMG. [Dissertação de Mestrado]. Minas Gerais: Faculdade de Odontologia da UFMG; 2005.

24. Santana JP, Campos FE, Sena RR. Formação profissional em saúde: desafios para a universidade. Texto de apoio elaborado especialmente para o Curso de Especialização em Desenvolvimento de Recursos Humanos de Saúde - CDRHU 1999 [citado 2010 set 17]. Disponível em: URL: HTTP://www.opas.org. $\mathrm{br} / \mathrm{rh} /$ publicacoes/textos_apoio/ACF2114.pdf

25. Secco LG, Pereira MLT. A profissionalização docente e os desafios político-estruturais dos formadores em odontologia. Revista da ABENO, 2004; 4(1): 22-8.

26. Segura MEC, Soares MS, Jorge WA. Programas extramuros nas instituições de ensino de odontologia na América Latina e nos Estados Unidos da América: contribuição ao estudo. Educación Médica y Salud, Washington, 1995; 29(2): 218-27.

Recebido em 07/07/2011 Aceito em 25/07/2011 